\section{Remote working solutions for your practice}

Now more than ever, remote working solutions are vital. Yo Telecom is here to help.

Yo Telecom is setting up virtual receptions for dental practices. This will allow you and your staff to take calls, have telephone triage, and arrange emergency appointments. Yo Telecom will provide softphones on your mobiles and laptops, meaning you can make and take calls through the main number of your practice. You can also transfer calls to your dentists for emergency cases, and retrieve all answer phone messages left from patients.

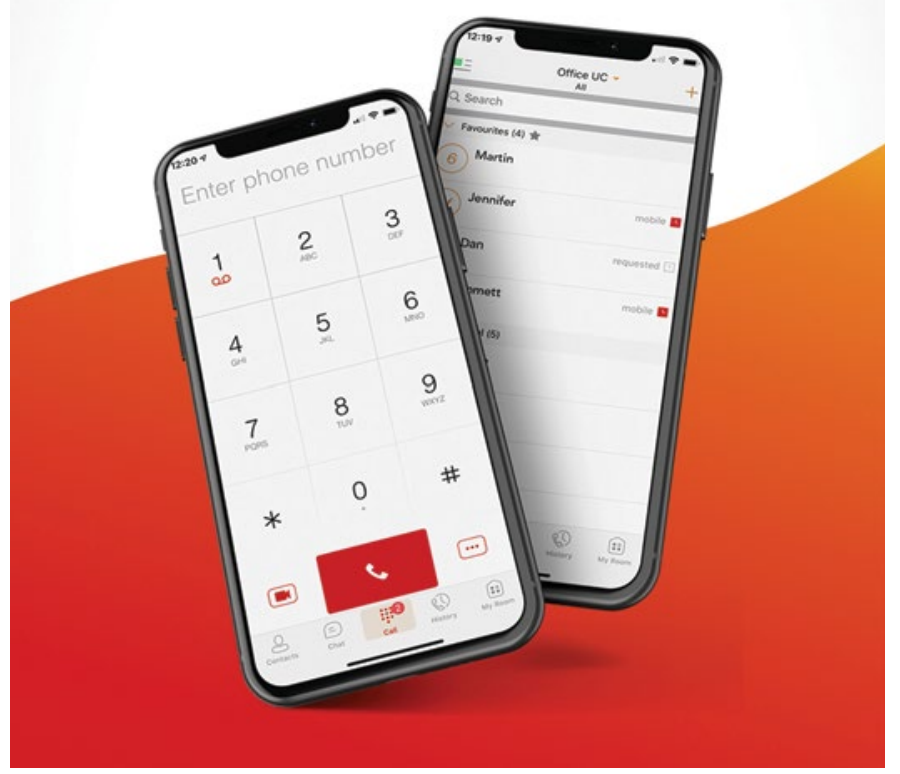

You will also be able to access your dental database and reception computers from your home. Yo Telecom's remote access software enables you to use your practice's computers from anywhere in the world. This will ensure that during these times, your quality of service remains high and you have access to all of your patients' information.

Get in touch now to find out how Yo Telecom can help you on 02381733966.

\section{Total protection for your practice}

When it comes to the transmission of harmful pathogens, dental practices are one of the most contaminated working environments in the world. The risk of cross infection is particularly high due to the dispersal of aerosols within the surgery caused by the use of high speed turbines in common treatments.

\section{AIRGENE CE Airborne} Disinfectant from Eschmann helps minimise this risk, enhancing existing hygiene processes and only taking one hour to ensure a 'belt and braces' approach to the daily decontamination regime.

AIRGENE CE's 'one shot' valve which automatically releases the high-level disinfectant achieves a dual effect: effective decontamination of micro-organisms over a wide area and elimination of bad odours caused by bacterial decomposition.

Just one $50 \mathrm{ml}$ canister covers an area of up to $40 \mathrm{~m} 3$, treating all surfaces, especially hard to reach and inaccessible places.

For use in dental surgeries and patient areas, AIRGENE CE should be used on a weekly basis or as frequently as required.

For more information on the highly effective and affordable range of decontamination equipment and products from Eschmann, visit www.eschmann.co.uk or call 01903875787.

\title{
Cement veneers effortlessly
}

G-CEM Veneer is a super-strength light-cure resin cement with the perfect consistency for luting extremely thin veneers with ease.

This aesthetic cement is made using one of the most innovative filler technologies available - Full-coverage Silane Coating (FSC).

A silane coupling agent which almost fully coats the fillers creates a strong connection with the matrix, which enables a greater filler rate of $69 \%$ within the cement and consequently delivers an ideal thixotropic consistency.

The result is a cement which stays in place when applied, but is fluid when under pressure. In this way, the entire surface of the restoration is uniformly covered without voids, whilst the film thickness is kept to a minimum. Crucially, G-CEM Veneer is not sticky either, so it is easy to apply precisely, with any excess being easily wiped away.

The high filler rate also gives it exceptional resistance to wear, enables a strong bond and provides excellent flexural strength, making it a strong and durable fixer for veneers, inlays and onlays with a thickness of $<2 \mathrm{~mm}$. Its translucency enables complete light-curing of the cement, making placement easier and faster than possible with other materials.

G-CEM Veneer has a natural-looking fluorescence and comes in four shades which blend in to cover all aesthetic needs. Its high conversion rate after light-curing also enables it to resist discolouration and staining over time, ensuring the restoration retains its aesthetic qualities in the long term.

Working with thin indirect restorations such as veneers, inlays and onlays has always been a challenge for restorative clinicians. G-CEM Veneer makes it so much easier.

To find out more contact GC UK Ltd on 01908 218999, email Info.uk@gc.dental or visitwww.gceurope.com. 